\title{
Mikroskop-basierter interferometrischer Zeilensensor zum schnellen Scannen optischer Funktionsflächen
}

\author{
P. Kühnhold ${ }^{1}$, H. Knell ${ }^{1}$, P. Lehmann ${ }^{1}$, S. Laubach ${ }^{2}$, G. Ehret ${ }^{2}$ \\ ${ }^{1}$ Fachgebiet Messtechnik, Fachbereich Elektrotechnik/Informatik, \\ Universität Kassel, Wilhelmshöher Allee 71, 34121 Kassel \\ Tel.: 0561-8046517, E-Mail: peter.kuehnhold@uni-kassel.de \\ ${ }^{2}$ Arbeitsgruppe Form- und Wellenfrontmetrologie, Physikalisch-Technische \\ Bundesanstalt, Bundesallee 100, 38116 Braunschweig
}

\section{Kurzfassung}

Die Form optischer Funktionsflächen, z. B. asphärischer Linsen, mit der erforderlichen Genauigkeit zu messen stellt die moderne Messtechnik vor große Herausforderungen. Die Hauptaufgabe dabei besteht darin, große Flexibilität, hohe Messgenauigkeit und hohe Messgeschwindigkeiten mit vertretbaren Gerätekosten zu vereinen.

In diesem Beitrag soll ein neues Messsystem vorgestellt werden, welches in der Lage ist, mit hoher Datenrate, kontaktlos und mit interferometrischer Genauigkeit die Formabweichung optischer Funktionsflächen zu erfassen. Verwendet wird dazu ein Michelson-Interferometermodul, welches mit einem Mikroskop mit 5x Vergrößerung kombiniert wird. Dieses Messsystem ist mit einer schmalbandigen Lichtquelle und einer Zeilenkamera ausgestattet und liefert 790000 Messpunkte pro Sekunde. Um die Interferenzphase zu erhalten, wird der Referenzspiegel zu einer Oszillation entlang der optischen Achse angeregt. Es resultieren phasenmodulierte Interferenzsignale, die ausgewertet und in Höhenwerte umgerechnet werden. Dieser interferometrische Zeilensensor wird mit geeigneten Postionier- und Scanachsen kombiniert, so dass die Formabweichung optischer Funktionsflächen erfasst werden kann. 


\section{Einleitung}

Interferometrische Messsysteme haben in den letzten Jahren zunehmend an Bedeutung gewonnen. Ihre Fähigkeit, Abstände schnell und kontaktlos mit einer Genauigkeit im Submikrometer Bereich auch bei größeren Messfeldern zu messen, zeichnet sie gegenüber anderen Verfahren (konfokale Messung, Deflektometrie) aus [1].

Flächenhaft messende Interferometer, die in der Regel nach dem Phasenschiebeverfahren arbeiten, erzielen zwar hohe Messgeschwindigkeiten, sind aber oft unflexibel in Bezug auf unterschiedliche Messobjekte. Flexible, punktförmig messende Interferometer lassen sich hingegen gut in Koordinatenmessmaschinen integrieren, benötigen aber vergleichsweise lange Messzeiten. Als Kompromiss wird in diesem Beitrag eine Zeilenkamera verwendet und ein Messprozess realisiert, der eine spezielle Art des Subapertur-Stitching darstellt [2].

Im Folgenden wird ein Messsystem vorgestellt, mit dem optische Funktionsflächen, z. B. asphärische Linsen (Asphären), mit interferometrischer Genauigkeit effizient gemessen werden können. Das Messsystem wurde in einem Kooperationsprojekt zwischen der Physikalisch-Technischen Bundesanstalt und der Universität Kassel realisiert. Die PTB stellt ein 5-Achsen-Bewegungssystem zur Verfügung, das mit dem interferometrischen Sensor kombiniert wird. Außerdem erarbeitet die PTB den Messablauf und ein geeignetes Stichting Verfahren für die 3D-Messdaten. Die Universität Kassel ist für die Auslegung, Realisierung, Ansteuerung und Signalauswertung des interferometrischen Zeilensensors verantwortlich.

\section{Auslegung des Messsystems}

In diesem Abschnitt sollen die in Frage kommenden Interferometertypen mit ihren Vor- und Nachteilen vorgestellt und diskutiert werden. Außerdem wird auf Realisierungsmöglichkeiten einer Phasenmodulation des Messsignals eingegangen. 


\subsection{Wahl der Interferometerbauart}

Für das Messsystem kommen sowohl Linnik- als auch Michelson-Interferometer in Frage. Beide Interferometerarten wurden aufgebaut und hinsichtlich ihrer Genauigkeit und praktischen Verwendbarkeit in Bezug auf Justageaufwand und Baugröße untersucht.

Ein Aufbau nach Linnik, bei dem zwei identische Mikroskopobjektive Verwendung finden, kann als optisch perfekte Anordnung betrachtet werden, weil keine optischen Elemente zwischen Objektiv und Messobjekt im Messarm eingefügt werden müssen und die Aufteilung zwischen Mess- und Referenzarm im afokalen Raum des Mikroskopsystems erfolgt. Außerdem steht der volle Arbeitsabstand der Objektive zur Verfügung. Nachteilig wirken sich die aufwendige Justage und die vergleichsweise hohen Kosten aus. Bild 1a) zeigt einen schematischen Aufbau eines Linnik-Interferometers, wobei die Mikroskopobjektive als einfache Linsen dargestellt sind.

Beim Michelson-Interferometer hingegen muss ein Strahlteiler zwischen Objektiv und Messobjekt eingebracht werden. Dies verringert den zur Verfügung stehenden Arbeitsabstand deutlich, wie der in Bild 1b) dargestellte schematische Aufbau zeigt. Zusätzlich erfährt das durch den Strahlteiler hindurchtretende Licht eine zweifache Brechung. Wird dies beim Design des Objektives nicht berücksichtigt, ist insbesondere bei Verwendung von Weißlicht mit Dispersionsfehlern zu rechnen. Dispersionsbedingte systematische Messfehler können zu $2 \pi$-Sprüngen bei der Auswertung der Phase des Messsignals führen, was sich in der gemessenen Oberflächentopographie in Höhenstufen von Vielfachen der halben mittleren Lichtwellenlänge niederschlägt [3,4]. Um diesen Messfehlern vorzubeugen, wurde eine Möglichkeit gefunden, die Effekte, die durch den Strahlteilerwürfel hervorgerufen werden, weitgehend zu kompensieren [4, 5]. Durch das Einbringen einer geeigneten Linsenkombination vor und hinter dem Strahlteilerwürfel, erleiden die Strahlen des auf die optische Achse fokussierten Lichtkegels keine Brechung mehr beim Durchtritt durch den Strahlteilerwürfel. Das auf diese Weise optisch korrigierte Interferometer ist in Bild 2 schematisch dargestellt. Mit dieser Korrektur ist es möglich, eine Modulationsübertragungsfunktion zu erreichen, die nahezu der eines Linnik-Interferometers entspricht, 
obwohl das Einbringen des Strahlteilerwürfels im Design des kommerziellen Objektives nicht berücksichtigt ist. Während sich das Linnik-Interferometer aufgrund der vielen benötigten Freiheitsgrade als sehr justageaufwendig erweist, ist die Justage des Michelson-Interferometers deutlich einfacher, denn es wird nur eine Linearverstellung für den Referenzspiegel benötigt [5].

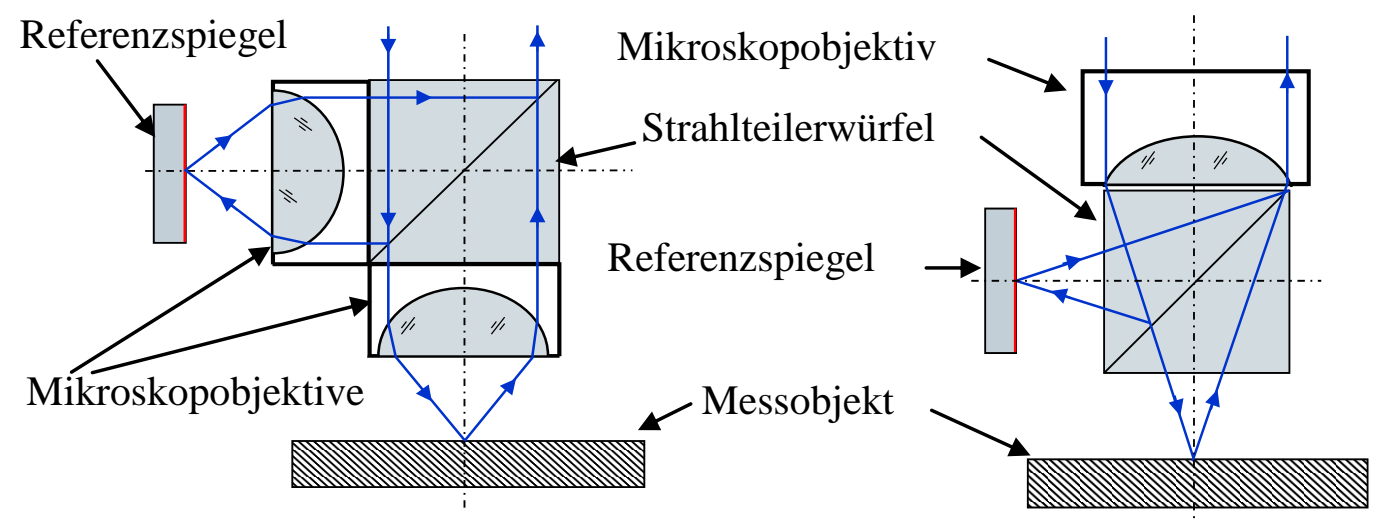

a)

b)

Bild 1: a) Schematischer Aufbau eines Linnik-Interferometers mit zwei identischen, auf unendliche Bildweite korrigierten Mikroskopobjektiven,

b) Schematischer Aufbau eines Michelson-Interferometers mit einem auf unendliche Bildweite korrigierten Mikroskopobjektiv.

\subsection{Phasenmodulation der Interferenzsignale}

Um die Phasenlage der Interferenzsignale bestimmen zu können, müssen diese zunächst phasenmoduliert werden. Dies wurde mit zwei unterschiedlichen Methoden erreicht. Die erste Methode besteht darin, das Messobjekt in Bezug auf die optische Achse des Interferometers um einen definierten Winkel zu kippen. Dadurch werden Interferenzstreifen als räumlich phasenmodulierte Trägerfrequenzsignale hervorgerufen [1, 6]. Die zweite Methode, die bei den im Folgenden dargestellten Ergebnissen verwendet wird, besteht darin, den Referenzspiegel mit Hilfe eines Piezoaktors in Schwingungen entlang der optischen Achse zu versetzen. Durch den schwingenden Referenzspiegel entstehen zeitlich phasenmodulierte Interferenzsignale [7]. In diesem Fall kann die Phaseninformation durch eine Demodulation auf Basis der Diskreten Fourier Transformation (DFT) bestimmt werden. Bei bekanntem Phasenverlauf ergibt sich der zugehörige Höhenverlauf durch Unwrapping. 


\subsection{Sensorkonfiguration}

Der Zeilensensor basiert auf einem selbstkonstruierten Michelson Interferenzaufsatz, der auf ein plan-apochromatisch, auf unendliche Bildweite korrigiertes 5x-Mikroskopobjektiv mit einer numerischen Apertur von 0,14 und einem Arbeitsabstand von $34 \mathrm{~mm}$ aufgeschraubt wird. Eine Tubuslinse erzeugt auf der CCD-Zeile eine optische Abbildung einer $2 \mathrm{~mm}$ langen Linie in der Objektebene. Die Kamera umfasst 1024 Pixel der Größe 10 x $10 \mu \mathrm{m}^{2}$ und kann mit einer maximalen Zeilenrate von $53 \mathrm{kHz}$ betrieben werden. Als Lichtquelle dient eine grüne LED, deren Kohärenzlänge durch ein Bandpassinterferenzfilter auf ca. 28 $\mu \mathrm{m}$ erhöht wird [8]. Verwendet man eine Weisslichtquelle in Verbindung mit einem Piezo-Hubtisch zur Durchführung des Tiefenscans lässt sich das Interferometer auch als konventionelles Weißlichtinterferometer betreiben.

Zur Phasenmodulation wird der Referenzspiegel mittels eines handelsüblichen Piezoaktors bewegt. Wird der Piezoaktor mit einer sinusförmigen Wechselspannung einer Frequenz von $390 \mathrm{~Hz}$ angeregt, resultiert eine Oszillationsamplitude $a$ von ca. $1 \mu \mathrm{m}$. Damit durchläuft das Interferenzsignal eines jeden Kamerapixels während einer halben Aktorperiode $n_{0}=2 a /(\lambda / 2)=4 a / \lambda \approx 7,5$ Perioden, die durch 64 zeitlich äquidistante Abtastwerte abgetastet werden. Dies ist ausreichend, um die Phasenlage des Interferenzsignals während einer halben Periode des Aktorsignals zuverlässig zu bestimmen, so dass man pro Periode des Aktorsignals für jedes Kamerapixel zwei Höhenmesswerte erhält. Als Anzahl $N_{\mathrm{ps}}$ der gemessenen Höhenwerte pro Sekunde folgt also: $N_{\mathrm{ps}}=390 \cdot 2 \cdot 1024 / \mathrm{s} \approx 790000 / \mathrm{s}$.

Für ein präzises Messergebnis ist eine möglichst perfekte Synchronisation des Aktorsignals mit dem Triggersignal der Kamera entscheidend. Um dies zu erreichen, nutzen beide Signale denselben Quarztakt, der von einem MikroController erzeugt wird.

\section{Messablauf und Signalauswertung}

Als Messobjekt dient zunächst eine plane Scheibe mit rotationssymmetrischer, umlaufender Sinusstruktur, die eine Amplitude von 0,25 $\mu \mathrm{m}$ und eine Perioden- 
länge von $100 \mu \mathrm{m}$ aufweist und in der PTB durch Ultrapräzisionszerspanung hergestellt wurde.

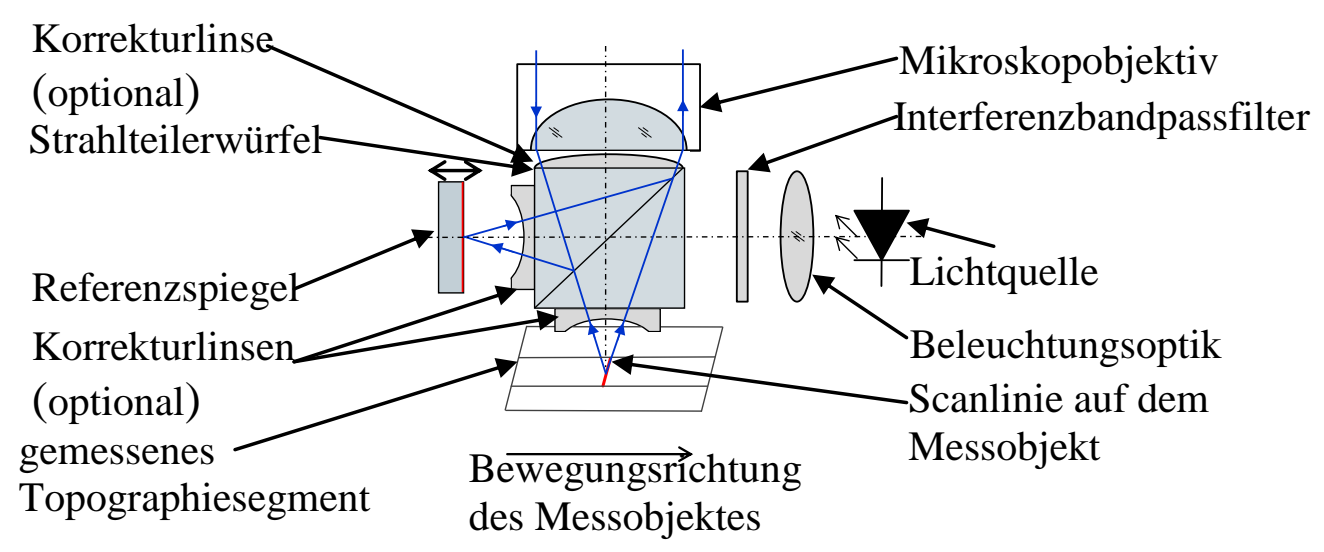

Bild 2: Schematischer Aufbau des Michelson-Interferometers mit drei Korrekturlinsen zur Wellenfrontanpassung, oszillierendem Referenzspiegel und der auf die Zeilenkamera abgebildeten Scanlinie.

\subsection{Messablauf}

Die Scheibe wird auf einem Drehtisch montiert und unter Zuhilfenahme des Interferometers zentriert. Anschließend wird das Messobjekt mit einem Autokollimator, dessen Licht in den Beleuchtungsstrahlengang des Interferometers eingekoppelt wird, so ausgerichtet, dass es gegenüber der optischen Achse des Interferometers keine Verkippung mehr aufweist.

Während der Messung wird das Messobjekt um $370^{\circ}$ gedreht. Dabei wird die Drehgeschwindigkeit sowohl an die Abtastrate der Kamera als auch an die laterale Auflösung des Interferometers angepasst. Die Überlappung von $10^{\circ}$ erleichtert die Vermeidung von Schließfehlern beim Messen von Kreisringsegmenten. Anschließend wird das Interferometer um die halbe Breite der durch das Interferometer gemessenen Linie in radialer Richtung verschoben und die Messung wird wiederholt [9].

\subsection{Signalauswertung}

Die Auswertung der Interferenzsignale erfolgt für jedes Kamerapixel der CCDZeile separat. Exemplarisch soll hier die Auswertung für ein Pixel erläutert werden. Um eine Messfrequenz von $780 \mathrm{~Hz}$ zu erreichen, ist sowohl bei steigender 
als auch bei fallender Flanke des Aktorsignals ein Höhenmesswert zu bestimmen. Ein typisches Interferenzsignal ist in Bild 3 dargestellt. Durch Anwendung einer Fensterfunktion werden zunächst die Bereiche, in denen die Signalfrequenz näherungsweise konstant ist, separiert. Durch eine Kalibriermessung an einem ruhenden Planspiegel wird die Signalfrequenz in diesen Bereichen ermittelt. Während der Messung werden die komplexen Fourierkoeffizienten der gefensterten Signale mittels DFT bei der durch die Kalibrierung ermittelten Signalfrequenz bestimmt. Daraus ergibt sich die Phasenlage der Signale mit hoher Genauigkeit. Diese Berechnung wird für alle Pixel für jede steigende und fallende Flanke durchgeführt. Anschließend werden die Phasenwerte in Höhenwerte umgerechnet. Um einen kontinuierlichen Topographieverlauf zu erhalten und gegebenenfalls auftretende Ausreißer in der Auswertung der Phase zu unterdrücken, wird ein 2D-Unwrapping durchgeführt [10]. Wird diese Prozedur auf ein umlaufendes Kreissegment angewendet, liegen anschließend die Höhenmesswerte für eine kreisringförmige Subapertur vor.

Solche Subaperturmessungen werden anschließend für unterschiedliche radiale Positionen durchgeführt und mit einem geeigneten Stitchingalgorithmus zusammengesetzt.

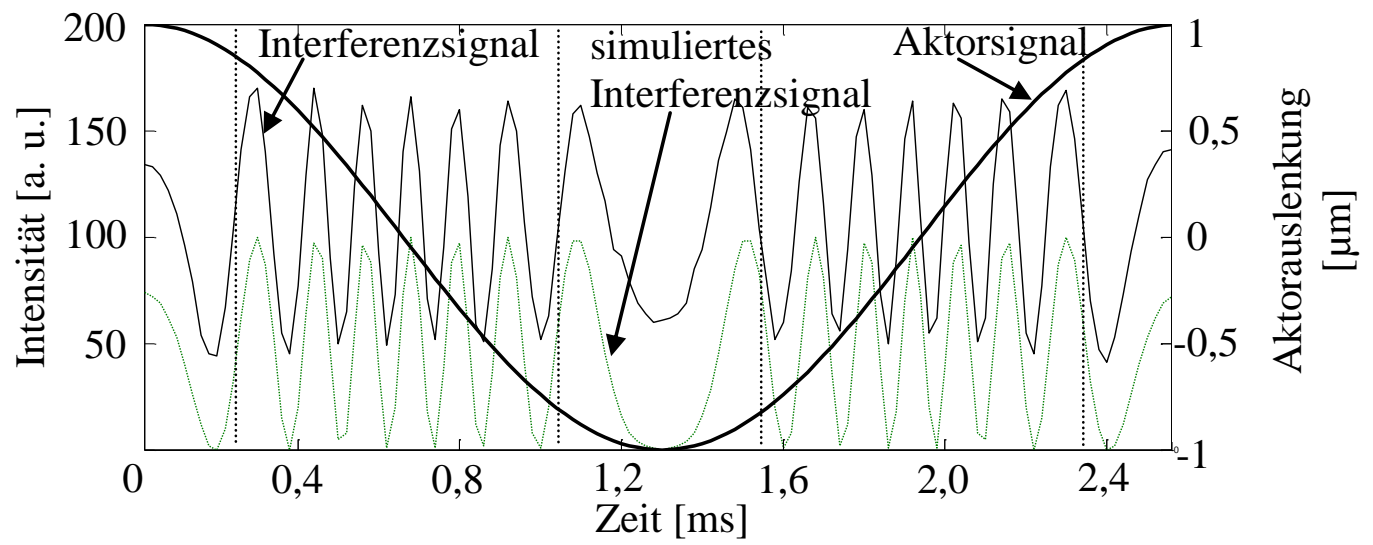

Bild 3: Gemessenes phasenmoduliertes Interferenzsignal (dünne Linie) eines Kamerapixels und das zugehörige simulierte Signal mit der derselben Anzahl an Abtastpunkten (gestrichelte dünne Linie) für eine Periode des ebenfalls dargestellten Aktorsignals (breite Linie), die gepunkteten senkrechten Linien markieren jeweils die Bereiche der fallenden bzw. steigenden Flanke, die zur Auswertung verwendet werden. 


\section{Messergebnisse}

Mit dem vorgestellten interferometrischen Sensor wurden bei Wiederholungsmessungen Standardabweichungen unter einem Nanometer erzielt. Um Topographien zu messen, wurde der Sensor mit zwei unterschiedlichen Scanachsen kombiniert: An der Universität Kassel wurde das Messobjekt mittels einer linearen $x$-Achse bewegt. Bild 4a) zeigt einen Ausschnitt eines solchen Messergebnisses für ein Sinusnormal mit $0,5 \mu \mathrm{m}$ Amplitude und $100 \mu \mathrm{m}$ Periodenlänge.

Bei der Physikalisch-Technischen Bundesanstalt diente ein Präzisons-Drehtisch als Scanachse. Bild 4b) zeigt ein Messergebnis für das in Abschnitt 3 beschriebene rotationssymmetrische Normal. Das dargestellte Resultat besteht aus zwei einzelnen Kreisringmessergebnissen, die durch einen Stitching-Algorithmus zusammengesetzt wurden.

Bild : 4 a) Messergebnis: Topographieausschnitt eines Sinusnormals mit einer Amplitude von $0,5 \mu \mathrm{m}$ und einer Periodenlänge von $100 \mu \mathrm{m}$,

b) Messergebnis: zwei separat gemessene Kreisringtopographien, die durch Stitching zu einer Gesamttopographie zusammengesetzt wurden.

\section{Zusammenfassung und Ausblick}

In diesem Beitrag wird ein linienförmig messender interferometrischer Sensor vorgestellt der für eine 1024 Pixel umfassende Linie von $2 \mathrm{~mm}$ Länge 780 Höhenmesswerte pro Sekunde und Pixel liefert. Der Sensor wertet die Phasenlage von Interferenzsignalen aus, die durch sinusförmige Weglängenmodulation im Referenzarm des Interferometers hervorgerufen werden. Er wurde sowohl 
mit einer linearen als auch mit einer rotatorischen Vorschubachse kombiniert und erfasst Abstandsänderungen präzise und zuverlässig.

Die Kombination des Sensors mit einer in der PTB vorhandenen Scan- und Positioniervorrichtung, die auf einem präzisen Drehtisch basiert, stellt alle Freiheitsgrade zur Verfügung, die zum Messen von komplexen rotationssymmetrischen Objekten wie asphärischen Linsen erforderlich sind.

Bedingt durch die numerische Apertur des verwendeten Objektives darf die maximale Neigung der lokalen Oberflächennormale des Messobjektes einen Winkel von $8^{\circ}$ gegenüber der optischen Achse des Sensors nicht überschreiten. Um auch Objekte mit größeren lokalen Neigungswinkeln messen zu können, soll künftig das auf dem Drehtisch befindliche Messobjekt, bzw. der Sensor mit Hilfe eines Kipptisches um definierte Winkel geneigt werden. Für eine Kalibierstrategie, die die Neigung des Messobjektes relativ zum Sensor berücksichtigt, ist ein absolutmessendes Interferometer vorteilhaft. Deshalb soll das Interferometer so erweitert werden, dass von der schmalbandigen LED-Beleuchtung auf eine Beleuchtung mit einer Weißlicht-LED umgeschaltet und das Interferometer als absolutmessendes Weißlichtinterferometer betrieben werden kann.

\section{Danksagung}

Für die Förderung dieses Projektes (LE 992/7-1, Eh 400/4-1) durch die Deutsche Forschungsgemeinschaft (DFG) bedanken sich die Autoren. Dieses Projekt ist eine Zusammenarbeit der Arbeitsgruppe Form- und Wellenfrontmetrologie der Physikalisch-Technischen Bundesanstalt (PTB) und des Fachgebiets Messtechnik am Fachbereich Elektrotechnik/Informatik der Universität Kassel.

\section{Literatur}

[1] Malacara, Daniel: Optical Shop Testing, John Wiley \& Sons Inc., Hoboken, 2007 
[2] Laubach, Sören, et al.: A Novel Form Measurement System for Precision Components using Interferometric Sub-aperture Stitching, Fringe 2013, 903 - 906, Springer, 2014

[3] Pavliček, Pavel, et al.: Measurement of the influence of dispersion on white light interferometry, App. Opt. Vol. 43 No. 4, 2004

[4] Lehmann, Peter, et al.: Reduction of chromatic aberration influences in vertical scanning white-light interferometry, Meas. Sci. Technol. 25, 065203 (9pp), 2014

[5] Kühnhold, Peter, et al.: Comparison of Michelson and Linnik interference microscopes with respect to measurement capabilities and adjustment efforts, Proc. SPIE 8788, 8788G, München, 2013

[6] Takeda, Mitsuo, et al.: Fourier-transform method of fringe pattern analysis for computer-based topography and interferometry, J. Opt. Soc. Am. Vol. 72 No. 1, 156-160, 1982

[7] Sasaki, Osami, et al.: Sinusoidal phase modulating interferometry for surface measurement, App. Opt. Vol. 25 No. 18, 3137-3140, 1985

[8] Knell, Holger, et al.: High speed measurement of specular surfaces on carrier fringe patterns in a line scan Michelson interferometer setup, Proc. SPIE 8788, 87880R, München, 2013

[9] Laubach, Sören, et al.: Stitching streifenförmiger Subaperturen zur Formmessung, Proc. DGaO, 2014, ISSN: 1614-8436

[10] Ghiglia, Dennis C., et al.: Two-Dimensional Phase Unwrapping, John Wiley \& Sons Inc., New York, 1998 